\title{
Cristiano Gori, Jose-Luis Fernandez and Raphael Wittenberg (eds.) (2016). Long-term Care Reforms in OECD Countries. Bristol: Policy Press, 316 pp. ISBN 9781447305057 (hardback)
}

\author{
REVIEWED by SHYH POH TEO*
}

Planning long-term care services is an important role for policy-makers and governments. This has generated much interest due to the increasing number of older people and the cost involved in providing services to clients in need of help and support. This book brings together international academic expertise to offer insight into the various approaches of OECD countries in managing this task. The lessons learnt are highly relevant to multiple disciplines, such as economics, political science, gerontology and sociology.

As a clinician involved in caring for older people, my experience is that providing recommendations for formal policies and service planning can be quite a daunting task. However, this book has given me a better understanding of the essential considerations necessary to put together effective long-term care systems. The authors manage to organise these complex details in an intuitive and easy-to-follow manner, providing a thought-provoking and accessible discussion of key components in

* Shyh Poh Teo, Geriatrics and Palliative Unit, Department of Internal Medicine, RIPAS Hospital, Brunei Darussalam 
International Journal of Ageing and Later Life

planning long-term care reforms. The chapters are sufficiently detailed to allow a novice reader to have a deeper understanding of each component; and for those who desire further specifics, references are available for further review.

The major considerations for long-term care, including funding, models of care, carers and institutional actors, are discussed systematically in this book. Each chapter is consistently laid out, beginning with an introduction covering the theoretical background for each concept. Comparisons are then made to contrast how selected OECD countries have tackled each aspect of long-term care. These are followed by an explanation of the rationale for each country's approach. General recommendations and concise key learning points are provided at the conclusion of each chapter.

The chapters detailing how different countries allocate long-term care resources to older clients, as well as how these have changed over time were quite fascinating and insightful. Detailed comparisons were made in terms of public funding versus client expenditure, universal coverage, means-testing or mixed systems. Specific countries utilising these different approaches were described, with observational cross-sectional data and longitudinal follow-up on how each country progressed over at least 15 years.

Although it was made clear to the reader what the expected outcomes were when different policy reforms were introduced, necessary adjustments made gradually over time were also explained. The unexpected trajectory changes were due to issues such as financial constraints or changes in preferences between offering services or cash. The reality is while long-term care policies are planned with the client's best interests in mind, real world challenges of increasing demand and service cost with scarcity of carers and client preferences necessitate fine-tuning of the programme. How this could be planned better from the start to minimise multiple incremental changes in retrospect could be lessons to reflect on, as these are useful experiences to learn from.

The main strength of this book is the critical thought process that went into comparisons between countries. Literature on long-term care usually describes individual countries in terms of their policies, taking into account which services are government funded or requires private spending and the 
Long-term Care Reforms in OECD Countries. Book Review

social circumstances of the population. There is variability in the range of services and care needs of each population, affected by historical, cultural or environmental factors. Therefore, comparison of data on long-term care between countries is challenging, especially to do so in a valid and standardised manner.

The book selected representative OECD countries with contrasting longterm care programmes and covered the strengths and weaknesses of each approach in a non-judgemental manner. The authors succinctly provided a synthesis of available information and pooled relevant observations from each country to distil objective explanations of why each system evolved the way it did. If consideration was given to concentrate more intensive services within institutions rather than home care, lessons are available from countries which tried this (e.g. Japan) versus those who preferred community-based services to increase coverage rate. The reader can then make a conscious choice through historical observation provided in the book to retrospectively decide what worked well or may backfire with the implementation of each approach.

The authors also showed significant foresight to include a chapter on integrated care. This relates to planning long-term care so that there is systematic coordination between social and health services. Often, integrated care only becomes a consideration after clients identify issues with fragmented care, when they struggle to navigate complex systems for both health care and long-term care providers. The fragmentation happens due to the separate development of specialised services, resulting in isolated silo work. There is opportunity to proactively reduce barriers between different types of services through careful planning from the start. The important components to achieve this are described, even though not many examples are available from the countries studied.

Although analysis was limited to OECD countries, this book is highly relevant to developing countries where long-term care is at its infancy. Despite the differences between OECD and developing countries, it is still useful to be armed with knowledge of the multi-dimensional view of each intervention in long-term care policies, leading to specific consequences and changes over time. The wide range of options to choose from can be adapted and applied to their current circumstance when starting from a 
International Journal of Ageing and Later Life

blank slate and unencumbered by inherited problems from previously introduced systems.

Overall, this book provides a comprehensive overview of long-term care policies in OECD countries. It provides a descriptive explanation of changes made over time for each country, with appropriate comparisons to make sense of observed trends. 